\title{
EFEITO DA ADIÇÃO DE CLORETOS METÁLICOS NA DECOMPOSIÇÃO TÉRMICA DE CASCA DE SOJA
}

\author{
J. A. SANTANA JÚNIOR ${ }^{1}$, C. H. ATAÍDE ${ }^{1}$ \\ ${ }^{1}$ Universidade Federal de Uberlândia, Faculdade de Engenharia Química \\ E-mail para contato: chataide@ufu.br
}

\begin{abstract}
RESUMO - A análise termogravimétrica, técnica analítica bastante utilizada para observar o comportamento térmico de materiais, foi utilizada para investigar a decomposição de casca de soja pura e com adição de cloretos metálicos. $\mathrm{O}$ foco principal deste trabalho foi realizar um estudo sobre decomposição térmica da casca de soja pura e com adição de $\mathrm{NaCl}, \mathrm{ZnCl}_{2}$ e $\mathrm{MgCl}_{2}$, podendo assim avaliar os efeitos provocados pela adição desses cloretos. As análises termogravimétricas foram realizadas em diferentes taxas de aquecimento $\left(5,10,15,20\right.$ e $\left.25^{\circ} \mathrm{C} / \mathrm{min}\right)$ em atmosfera inerte de nitrogênio, para a casca de soja pura e com adição de $\mathrm{NaCl}, \mathrm{ZnCl}_{2}$ e $\mathrm{MgCl}_{2}$ nas concentrações de 10 , 20 e $30 \%$. Inicialmente, observou-se um incremento na temperatura de degradação da amostra com o aumento da taxa de aquecimento. As curvas perda de massa e derivada de perda massa para a casca de soja com adição de sais evidenciaram uma redução da temperatura de degradação para as amostras com adição de 20 e $30 \%$ de $\mathrm{ZnCl}_{2}$ e todas as amostras com adição de $\mathrm{MgCl}_{2}$. Para as amostras com adição de $\mathrm{NaCl}$ houve um aumento da temperatura de degradação.
\end{abstract}

\section{INTRODUÇÃO}

Com a crescente preocupação com o aquecimento global devido as emissões de dióxido de carbono, a redução gradativa de fontes mais acessíveis dos combustíveis fósseis e o aumento da procura de um fornecimento sustentável de combustíveis, aumentaram o interesse em fontes limpas e renováveis de energia. Recursos renováveis oriundos da biomassa vegetal se tornam cada vez mais importantes como alternativa capaz de reduzir a dependência dos combustíveis fósseis

A casca da soja, objeto de estudo deste trabalho é classificado como resíduo agroindustrial e vem ganhando destaque devido ao aumento da disponibilidade deste resíduo no mercado. A casca de soja é um subproduto obtido da industrialização da soja, corresponde à fina camada que recobre o grão. A casca de soja representa cerca de $2 \%$ (em peso) do grão inteiro. A inclusão da casca de soja na alimentação animal tem sido a principal aplicação desse resíduo (Zambom et al., 2001).

Várias tecnologias capazes de converter a biomassa em energia têm sido cada vez mais estudadas. Uma tecnologia capaz converter a biomassa em produtos químicos e combustíveis líquidos mais valiosos será importante para atenuar alguns problemas ambientais e disponibilidade de energia (Murata et al., 2012). A pirólise vem se destacando como um processo eficiente de conversão termoquímica de biomassa, a temperatura relativamente moderadas $\left(300-600{ }^{\circ} \mathrm{C}\right)$, em outros produtos (Murata et al., 2012). Os produtos oriundos da pirólise podem ser classificados em três grandes 


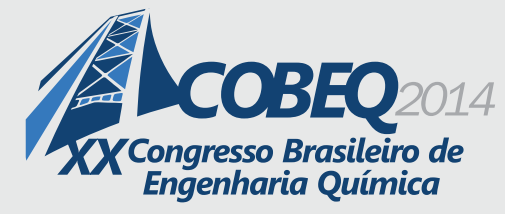

categorias com base no seu estado físico: bio-óleo (líquido), carvão (sólido) e gases não condensáveis. O bio-óleo é uma mistura que pode conter mais de 400 diferentes compostos, incluindo ácidos, alcoóis, aldeídos, ésteres, cetonas e compostos aromáticos (Huber et al., 2006).

Para uma melhor compreensão dos processos de conversão de diversas biomassas, é comum investigar o comportamento térmico, através da decomposição desses materiais por análise termogravimétrica. A análise termogravimétrica é um método de alta precisão para o estudo da pirólise, em condições bem definidas ( $\mathrm{Li}$ et al., 2013). Essa análise fornece informações indispensáveis para o desenvolvimento de novas tecnologias, como a pirólise (Buryan e Staff, 2008).

Adição de aditivos ou catalisadores confere ao processo maior flexibilidade para regular ou ajustar o processo de pirólise. Como existe uma enorme variedade de biomassas, com estruturas e componentes bastante diferentes, a pirólise de materiais distintos certamente necessitará de aditivos ou catalisadores igualmente diferentes na etapa de desenvolvimento e otimização. A análise térmica pode fornecer uma avaliação preliminar rápida de efeitos catalíticos de aditivos na pirólise. A perda de peso da amostra em função do tempo e temperatura é fornecida nesta técnica e, com esses resultados pode-se facilmente avaliar o efeito dos catalisadores sobre o processo de pirólise de biomassa. Além disso, este método tem a vantagem de utilizar uma quantidade muito pequena de amostras (Chattopadhyay et al., 2009).

Por razões econômicas, muitos pesquisadores defendem que o futuro da pirólise rápida da biomassa lignocelulósica, está na produção de químicos importantes e não propriamente na obtenção do bio-óleo. Nesse caso, a conversão da biomassa em produtos aromáticos, por exemplo, deve acontecer na presença de catalisadores. Altas taxas de aquecimento e elevadas razões catalisador/biomassa no reator, favorecem a produção de compostos aromáticos (Carlson et al. 2010).

Segundo Chalov et al. (2014) a atividade catalítica de cloretos metálicos incrementa com o aumento de sua acidez relativa. Os catalisadores de cloretos metálicos podem divididos em três grupos (Chalov et al., 2014): catalisadores com baixa acidez ou eles não estão ativos na processo de pirólise $(\mathrm{KCl}, \mathrm{NaCl})$; catalisadores com média acidez, possuem atividade e seletividade relativamente elevada $\left(\mathrm{NiCl}_{2}, \mathrm{CoCl}_{2}, \mathrm{ZnCl}_{2}, \mathrm{FeCl}_{2}\right)$; catalisadores com alta acidez, possuem alta atividade mas baixa seletividade $\left(\mathrm{MgCl}_{2}, \mathrm{FeCl}_{3}, \mathrm{AlCl}_{3}\right)$. Nesse trabalho para realizar um estudo com adição de cloretos de diferentes acidez foi escolhido um cloreto de baixa acidez $(\mathrm{NaCl})$, um de média acidez $\left(\mathrm{ZnCl}_{2}\right)$ e um de alta acidez $\left(\mathrm{MgCl}_{2}\right)$.

Para uma melhor identificação da biomassa utilizada foram determinadas algumas de suas propriedades, tais como análise elementar, composição química, análise imediata e poder calorífico superior. Outras propriedades físicas relevantes, tais como densidade, distribuição de tamanho, razão de aspecto e esfericidade foram investigadas em um trabalho anterior (Cardoso et al., 2013).

O presente trabalho teve como objetivo principal realizar um estudo da decomposição térmica da casca de soja pura e com adição de $\mathrm{NaCl}, \mathrm{ZnCl}_{2}$ e $\mathrm{MgCl}_{2}$. Para isso, foi necessária a obtenção de dados experimentais de decomposição térmica, obtidos através da análise termogravimétrica. 


\section{EXPERIMENTAL}

\subsection{Materiais}

A casca de soja utilizada nesse estudo foi cedida pela empresa Cargill Agrícola S/A - Unidade Uberlândia. A Tabela 1 mostra os resultados de análise elementar, composição química, análise imediata e poder calorífico superior da amostra de casca de soja.

Tabela 1 - Características da casca de soja

\begin{tabular}{|c|c|c|c|c|c|c|}
\hline \multicolumn{2}{|c|}{$\begin{array}{c}\text { Análise } \\
\text { elementar }(\%)\end{array}$} & \multicolumn{2}{|c|}{ Composição química (\%) } & \multicolumn{2}{|c|}{ Análise imediata (\%) } & \multirow[t]{2}{*}{$\begin{array}{l}\text { Poder calorífico } \\
\text { superior }(\mathrm{MJ} / \mathrm{Kg})\end{array}$} \\
\hline $\mathrm{C}$ & 39,27 & Holocelulose & 80,56 & Material volátil & 79,02 & \\
\hline $\mathrm{H}$ & 5,76 & Lignina & 4,83 & Cinzas & 5,22 & \\
\hline $\mathrm{N}$ & 1,96 & Extrativos & 14,61 & $\begin{array}{l}\text { Umidade (base } \\
\text { úmida) }\end{array}$ & 7,75 & 17,73 \\
\hline $\mathrm{S}$ & 0,06 & & & Carbono fixo & 8,01 & \\
\hline $\mathrm{O}$ & 52,95 & & & & & \\
\hline
\end{tabular}

Os cloretos metálicos utilizados na impregnação da biomassa, $\mathrm{NaCl}(\geq 99 \%), \mathrm{ZnCl}_{2}(\geq 98 \%)$ e $\mathrm{MgCl}_{2}(\geq 99 \%$ ), foram obtidos da Sigma Aldrich. As amostras de biomassa foram impregnadas com os cloretos em concentrações de 10, 20 e $30 \%$ (peso).

\subsection{Métodos}

As análises termogravimétricas para as amostras de casca de soja pura e impregnada foram realizadas em um analisador termogravimétrico Shimadzu, modelo TGA/DTG-60H. Medições não isotérmicas foram realizadas sob fluxo contínuo de atmosfera inerte de nitrogênio gasoso de alta pureza, a taxa de $30 \mathrm{~mL} / \mathrm{min}$. Amostras de aproximadamente $14 \mathrm{mg}$ foram utilizadas nas análises.

Foram realizados experimentos dinâmicos nos quais o material foi aquecido a $100{ }^{\circ} \mathrm{C}$, com taxa de aquecimento de $50{ }^{\circ} \mathrm{C} / \mathrm{min}$, e mantido a essa temperatura por 30 minutos, para eliminar a umidade. Logo após, o material foi aquecido a $900{ }^{\circ} \mathrm{C}$ empregando cinco diferentes taxas de aquecimento: 5 , $10,15,20$ e $25^{\circ} \mathrm{C} / \mathrm{min}$ (cada experimento foi realizado três vezes). Os dados referentes aos primeiros 30 minutos foram desconsiderados, assim as variações de massa devido à perda de água não foram consideradas. As análise foram realizadas nas mesmas condições utilizadas por Cardoso et al. (2011).

\section{RESULTADOS E DISCUSSÃO}

As curvas perdas de massa ou TG (visualizada na escala da esquerda) e derivada de perda massa ou DTG (visualizada na escala da direita) para a casca de soja em diferentes taxas de aquecimento são mostradas na Figura 1. Nessa figura fica claro o efeito da taxa de aquecimento na decomposição 


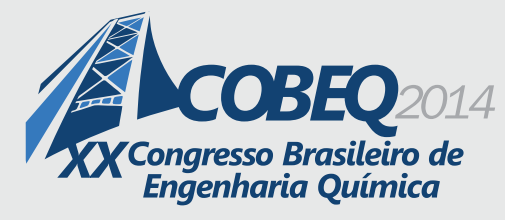

19 a 22 de outubro de 2014

Florianópolis/SC

térmica da casca de soja. A taxa de aquecimento modifica as posições da curva TG e DTG, além disso, ocorreu um aumento na amplitude do pico DTG, indicando um aumento da taxa de degradação máxima. Com o aumento da taxa de aquecimento a decomposição passa a ocorrer em temperaturas mais altas, esse fato é reportado na literatura para outras biomassas, como em Damartzis et al. (2011), Slopiecka et al. (2012) e Ounas et al. (2011).

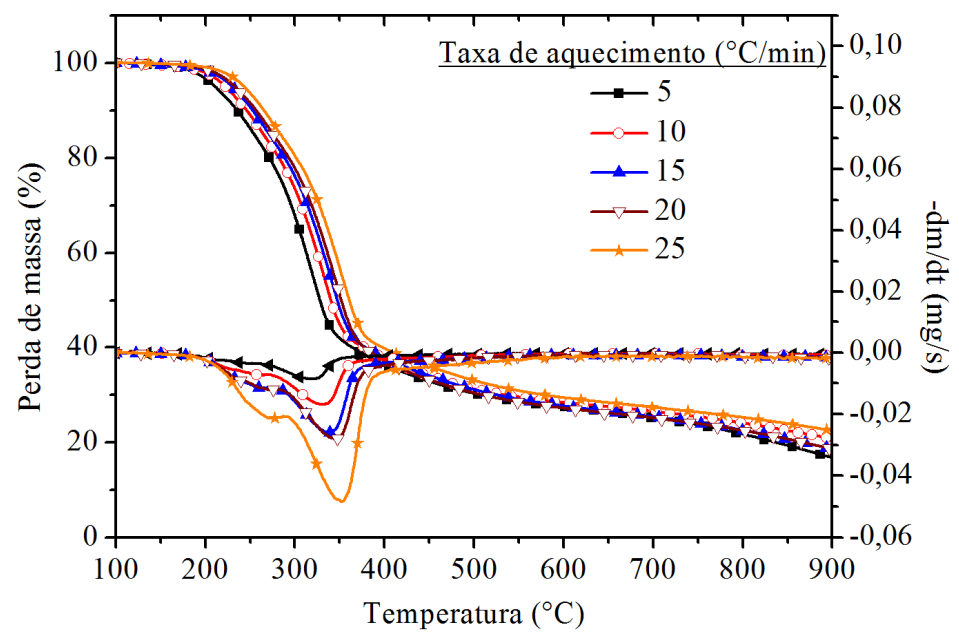

Figura 1 - Curvas TG e DTG para a casca de soja.

As Figuras 2, 3 e 4 mostram os resultados das análises termogravimétricas para a casca de soja com adição de $\mathrm{NaCl}, \mathrm{ZnCl}_{2}$ e $\mathrm{MgCl}_{2}$, respectivamente, para taxa de aquecimento de $15{ }^{\circ} \mathrm{C} / \mathrm{min}$. As análises foram realizadas em cinco taxas de aquecimento, e pode-se constatar o mesmo efeito causado pelo aumento da taxa de aquecimento observado nas curvas para a casca de soja pura. Os outros resultados das análises termogravimétricas da biomassa com os sais, para as demais taxas de aquecimento $\left(5,10,20\right.$ e $\left.25^{\circ} \mathrm{C} / \mathrm{min}\right)$, apresentaram comportamentos bastante similares. A Tabela 2 mostra como a temperatura inicial de degradação é modificada com a adição dos cloretos em diferentes concentrações e várias taxas de aquecimento. As temperaturas iniciais de degradação mostradas nessa tabela são referentes a perda de peso de $90-70 \%$ nas amostras, medidas a partir das curvas TG, seguindo a mesma faixa de perda de peso utilizada por Amarasekara e Ebede (2009).

$\mathrm{Na}$ Figura 2 pode ser observado que a adição de $\mathrm{NaCl}$ apresentou um pequeno efeito na degradação térmica da casca de soja. Em todas as condições analisadas é possível notar, nas curvas $\mathrm{TG}$, que o $\mathrm{NaCl}$ fez com que ocorra a desvolatização da casca de soja em temperaturas pouco maiores, seguindo a ordem de concentração de sal adicionada à biomassa. Esse efeito pode ser visualizado também na Tabela 2, com o incremento da concentração de $\mathrm{NaCl}$ adicionada, a temperatura inicial de degradação teve um pequeno aumento. As amostras contendo $30 \%$ de $\mathrm{NaCl}$, em diferentes taxas de aquecimento, apresentaram as maiores faixas de temperatura inicial de degradação. As curvas DTG indicam que as amostras impregnadas com $\mathrm{NaCl}$ reduziram as taxas máximas de degradação da biomassa proporcionalmente ao aumento do teor de cloreto de sódio presente na amostra. A Tabela 2 mostra também a tendência de aumento da temperatura de degradação com incremento da taxa de aquecimento, já comentada anteriormente. 


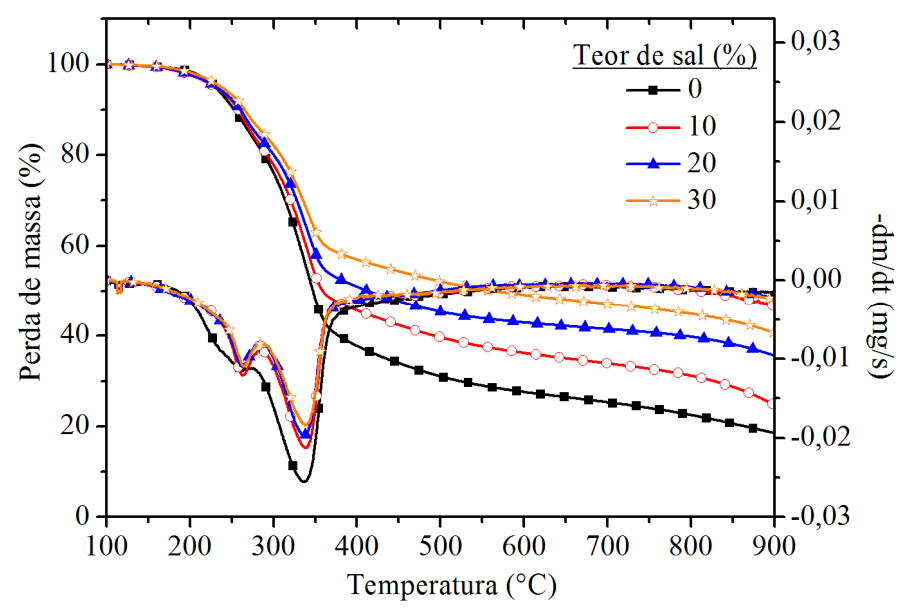

Figura 2 - Curvas TG e DTG para a casca de soja pura e impregnada com $\mathrm{NaCl}$ (taxa de aquecimento: $15^{\circ} \mathrm{C} / \mathrm{min}$ ).

A Figura 3 mostra o efeito provocado pela adição de diferentes concentrações de $\mathrm{ZnCl}_{2}$. É possível notar, na curva TG, que a adição de $10 \%$ de $\mathrm{ZnCl}_{2}$ teve pouco efeito sobre a desvolatização da biomassa. Já para concentrações maiores $(20$ e 30\%) do sal adicionado à biomassa a desvolatização ocorreu em temperaturas menores do que na biomassa pura. Na Tabela 2 verifica-se que com adição de $10 \%$ de $\mathrm{ZnCl}_{2}$, a temperatura de degradação teve, inicialmente, um pequeno aumento, seguida de uma pequena redução em relação a temperatura da amostra sem adição de $\mathrm{ZnCl}_{2}$. As amostras contendo 20 e $30 \%$ de $\mathrm{ZnCl}_{2}$ tiveram uma redução da temperatura inicial de degradação. A curvas DTG, na Figura 3, mostram que as amostras impregnadas com $\mathrm{ZnCl}_{2}$ reduziram as taxas máximas de degradação da biomassa conforme o aumento do teor de sal adicionado.

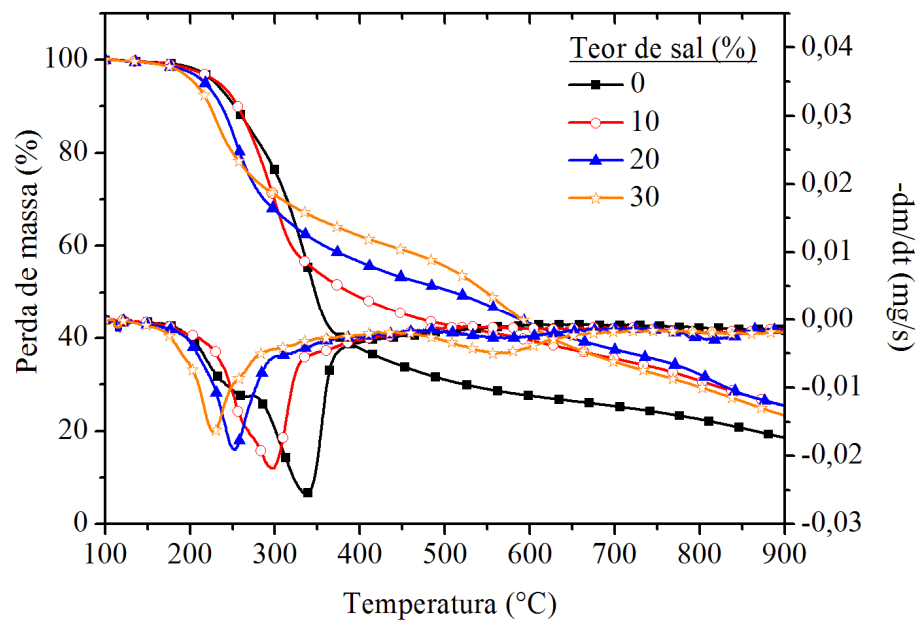

Figura 3 - Curvas TG e DTG para a casca de soja pura e impregnada com $\mathrm{ZnCl}_{2}$ (taxa de aquecimento: $\left.15^{\circ} \mathrm{C} / \mathrm{min}\right)$. 


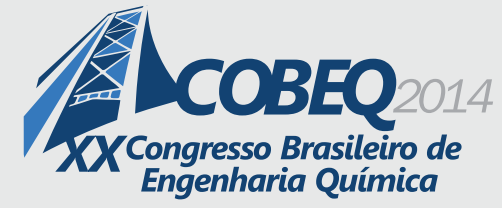

Tabela 2 - Mudança na faixa de temperatura inicial de degradação com a adição de sais a casca de soja.

\begin{tabular}{|c|c|c|c|c|}
\hline $\begin{array}{l}\text { Teor de } \\
\text { sal (\%) }\end{array}$ & $\begin{array}{c}\text { Taxa de } \\
\text { aquecimento } \\
\left({ }^{\circ} \mathrm{C} / \mathrm{min}\right) \\
\end{array}$ & $\begin{array}{l}\text { Faixa de temperatura } \\
\text { inicial de degradação } \\
\quad\left({ }^{\circ} \mathrm{C}\right) \text { com } \mathrm{NaCl}\end{array}$ & $\begin{array}{l}\text { Faixa de temperatura } \\
\text { inicial de degradação } \\
\quad\left({ }^{\circ} \mathrm{C}\right) \operatorname{com} \mathrm{ZnCl}_{2}\end{array}$ & $\begin{array}{l}\text { Faixa de temperatura } \\
\text { inicial de degradação } \\
\left({ }^{\circ} \mathrm{C}\right) \text { com } \mathrm{MgCl}_{2} \\
\end{array}$ \\
\hline \multirow{5}{*}{0} & 5 & $231-294$ & $231-294$ & $231-294$ \\
\hline & 10 & $242-306$ & $242-306$ & $242-306$ \\
\hline & 15 & $249-312$ & $249-312$ & $249-312$ \\
\hline & 20 & $253-317$ & $253-317$ & $253-317$ \\
\hline & 25 & $262-326$ & $262-326$ & $262-326$ \\
\hline \multirow{5}{*}{10} & 5 & $235-299$ & $239-283$ & $208-269$ \\
\hline & 10 & $246-310$ & $249-293$ & $218-279$ \\
\hline & 15 & 254-319 & 253-298 & $223-282$ \\
\hline & 20 & $257-323$ & $259-302$ & $227-285$ \\
\hline & 25 & $263-329$ & $265-307$ & $232-289$ \\
\hline \multirow{5}{*}{20} & 5 & $240-307$ & $222-273$ & $187-277$ \\
\hline & 10 & $248-317$ & $229-279$ & $194-279$ \\
\hline & 15 & $257-326$ & $235-283$ & $202-289$ \\
\hline & 20 & $260-330$ & $239-287$ & $206-292$ \\
\hline & 25 & $266-336$ & $242-289$ & $211-293$ \\
\hline \multirow{5}{*}{30} & 5 & $245-314$ & $208-285$ & $172-268$ \\
\hline & 10 & $254-326$ & $216-293$ & $187-280$ \\
\hline & 15 & $262-333$ & $221-300$ & $192-288$ \\
\hline & 20 & $265-338$ & $226-303$ & $196-290$ \\
\hline & 25 & $272-345$ & $229-309$ & $200-298$ \\
\hline
\end{tabular}

$\mathrm{Na}$ Figura 4 observa-se claramente o efeito provocado pela adição de $\mathrm{MgCl}_{2}$ na degradação térmica da casca de soja. Em todas as condições é possível notar, nas curvas TG, que o $\mathrm{MgCl}_{2}$ fez com que ocorresse a desvolatização da casca de soja em temperaturas menores do que na casca de soja pura, seguindo a ordem de concentração do cloreto adicionado à biomassa. $\mathrm{O}$ efeito provocado pela adição de $\mathrm{MgCl}_{2}$ na degradação térmica da casca de soja pode ser observado também na Tabela 2 , que mostra a mudança na temperatura inicial de degradação em diferentes taxas de aquecimento. A referida tabela mostra que com o aumento do teor de $\mathrm{MgCl}_{2}$ presente na amostra, houve uma redução da temperatura inicial de degradação. As amostras contendo $30 \%$ de $\mathrm{MgCl}_{2}$, em diferentes taxas de aquecimento, apresentaram as menores faixas de temperatura inicial de degradação. As curvas DTG mostradas na Figura 4, indicam que as amostras impregnadas com $\mathrm{MgCl}_{2}$ reduziram significativamente as taxas máximas de degradação da biomassa. Pode ser observado também que as curvas apresentam um deslocamento para a esquerda proporcional ao aumento da concentração de sal adicionado, demonstrando uma redução na temperatura de degradação. 


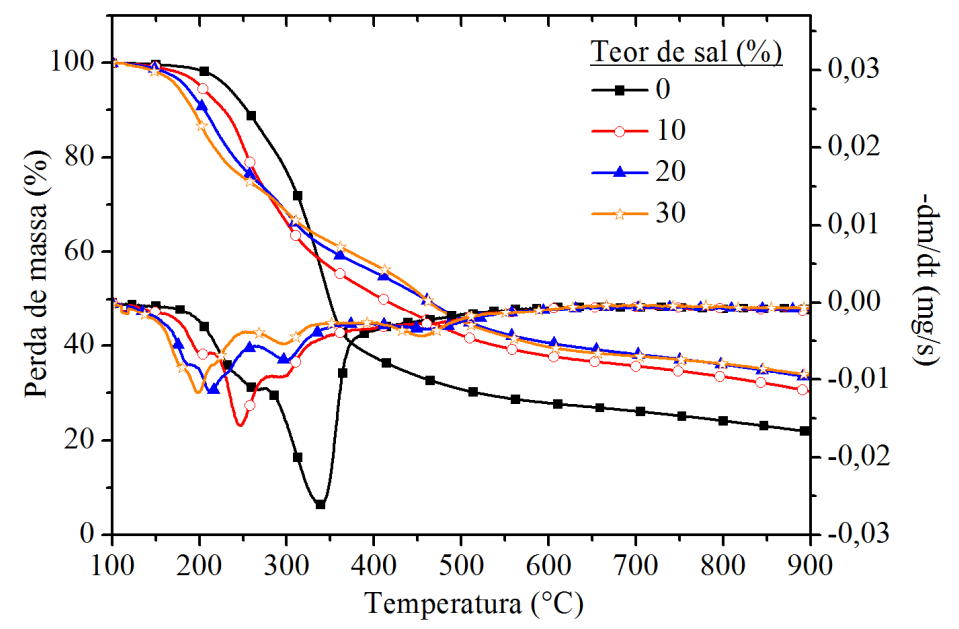

Figura 4 - Curvas TG e DTG para a casca de soja pura e impregnada com $\mathrm{MgCl}_{2}$ (taxa de aquecimento: $\left.15^{\circ} \mathrm{C} / \mathrm{min}\right)$.

\section{CONCLUSÕES}

As análises termogravimétricas da casca de soja pura e com adição de sais inorgânicos realizadas em diferentes taxas de aquecimento mostraram o efeito provocado pelo aumento da taxa de aquecimento. Em todos os casos, houve um aumento da temperatura de degradação com o incremento da taxa de aquecimento. As curvas DTG mostraram ainda que houve uma redução da taxa de degradação máxima com o incremento da taxa de aquecimento.

Através das curvas TG e DTG apresentadas, ficou claro o efeito provocado pela impregnação de cloretos metálicos na casca de soja.

Para as amostras de casca de soja com adição de $\mathrm{NaCl}$ houve um aumento da temperatura de degradação em todas as concentrações adicionadas. As curvas TG e DTG para a casca de soja com adição de sais indicaram que houve uma redução da temperatura de degradação para as amostras de casca de soja com adição de 20 e $30 \%$ de $\mathrm{ZnCl}_{2}$ e todas as amostras com adição de $\mathrm{MgCl}_{2}$. O efeito da redução de temperatura seguiu a seguinte ordem: $\mathrm{MgCl}_{2}>\mathrm{ZnCl}_{2}$, sequencia que segue a ordem de acidez dos cloretos. Esse resultado coincide com o reportado na literatura de que a atividade catalítica de cloretos metálicos incrementa com o aumento de sua acidez relativa.

\section{AGRADECIMENTOS}

Os autores agradecem a CAPES (Coordenacão de Aperfeiçoamento de Pessoal de Nível Superior), CEMIG (Companhia Energética de Minas Gerais) e FAPEMIG (Fundação de Amparo à Pesquisa do Estado de Minas Gerais) pelo apoio financeiro. Agradecemos a Fundação de Amparo à Pesquisa do Estado de Minas Gerais (FAPEMIG) pelos recursos concedidos no Projeto de Participação Coletiva em Eventos Técnicos-Científicos (PCE-00082-14). 


\section{REFERÊNCIAS}

AMARASEKARA A. S.; EBEDE C. C. Zinc chloride mediated degradation of cellulose at $200 \mathrm{C}$ and identification of the products. Bioresour. Technol., v. 100, p. 5301-5304, 2009.

BURYAN P.; STAFF M. Pyrolysis of the waste biomass. J. Therm. Anal. Calorim., v. 93, p. 637640, 2008.

CARDOSO C. R.; OLIVEIRA T. J. P.; SANTANA JUNIOR J. A.; ATAÍDE C. H. Physical characterization of sweet sorghum bagasse, tobacco residue, soy hull and fiber sorghum bagasse particles: Density, particle size and shape distributions. Powder Technol., v. 245, p. 105-114, 2013.

CARDOSO C. R.; MIRANDA M. R.; SANTOS K. G.; ATAÍDE C. H. Determination of kinetic parameters and analytical pyrolysis of tobacco waste and sorghum bagasse. J. Anal. Appl. Pyrolysis, v. 92, p. 392-400, 2011.

CARLSON T. R.; JAE J.; LIN Y. C.; TOMPSETT G. A.; HUBER G.W. Catalytic fast pyrolysis of glucose with HZSM-5: The combined homogeneous and heterogeneous reactions. J. Catal., v. 270, p. 110-124, 2010.

CHALOV K. V.; LUGOVOY Y. V.; DOLUDA V. Y.; SIDOROV A. I.; SULMAN M. G.; KOSIVTSOV Y. Y.; TKACHENKO O. P.; SULMAN E. M. Influence of metals chlorides on oilslime thermocatalytic processing. Chem. Eng. J., v. 238, p. 219-226, 2014.

CHATTOPADHYAY J.; KIM C.; KIM R.; PAK D. Thermogravimetric study on pyrolysis of biomass with $\mathrm{Cu} / \mathrm{Al}_{2} \mathrm{O}_{3}$ catalysts. J. Ind. Eng. Chem., v. 15, p.72-76, 2009.

DAMARTZIS T. H.; VAMVUKA D.; SFAKIOTAKIS S.; ZABANIOTOU A. Thermal degradation studies and kinetic modeling of cardoon (Cynara cardunculus) pyrolysis using thermogravimetric analysis (TGA). Bioresour. Technol., v. 102, p. 6230-6238, 2011.

HUBER G. W.; IBORRA S.; CORMA A. Synthesis of Transportation Fuels from Biomass: Chemistry, Catalysts and Engineering. Chem. Rev., v. 106, p. 4044-4098, 2006.

LI L.; WANG G.; WANG S.; QIN S. Thermogravimetric and kinetic analysis of energy crop Jerusalem artichoke using the distributed activation energy model. J. Therm. Anal. Calorim., v. 114, p.1183-1189, 2013.

MURATA K.; LIU Y.; INABA M.; TAKAHARA I. Catalytic fast pyrolysis of jatropha wastes. $J$. Anal. Appl. Pyrolysis, v. 94, p. 75-82, 2012.

OUNAS A.; ABOULKAS A.; EL HARFI K.; BACAOUI A.; YAACOUBI A. Pyrolysis of olive residue and sugar cane bagasse: Non-isothermal thermogravimetric kinetic analysis. Bioresour. Technol., v. 102, p. 11234-11238, 2011.

SLOPIECKA K.; BARTOCCI P.; FANTOZZI F. Thermogravimetric analysis and kinetic study of poplar wood pyrolysis. Appl. Energy, v. 97, p. 491-497, 2012.

ZAMBOM M. A.; SANTOS G. T.; MODESTO E. C.; AlCALDE C. R.; GONÇALVES G. D.; SILVA D. C.; SILVA K. T.; FAUSTINO J. O. Valor nutricional da casca do grão de soja, farelo de soja, milho moído e farelo de trigo para bovinos, Acta Sci., v. 23, p. 937-943, 2001. 